\title{
Diphtheria Outbreaks in Schools in Central Highland Districts, Vietnam, 2015-2018
}

Noriko Kitamura, Thao T.T. Le, Lien T. Le, Luong D. Nguyen, Anh T. Dao, Thanh T. Hoang, Keisuke Yoshihara, Makiko lijima, Tran M. The, Hung M. Do, Huy X. Le, Hung T. Do, Anh D. Dang, Mai Q. Vien, Lay-Myint Yoshida

During 2015-2018, seven schools in rural Vietnam experienced diphtheria outbreaks. Multilocus sequence types were the same within schools but differed between schools. Low vaccine coverage and crowded dormitories might have contributed to the outbreaks. Authorities should consider administering routine vaccinations and booster doses for students entering the school system.

$\mathrm{D}$ iphtheria is a serious childhood disease with a high mortality rate (1). After a diphtheria-tetanus-pertussis vaccine (DTP) was introduced in the early 20th century, the number of cases dramatically decreased. Incidence reached a low of 4,333 cases in 2006, but more recently, the number of reported cases has increased, with incidence reaching 16,648 cases in 2018 (2).

In 1981, Vietnam introduced a vaccination program in which participants received 3 primary doses of DTP (DTP3) vaccine; in 2011, a booster shot (DTP4) to be given 18 months after the initial doses was added (3). Although diphtheria cases had become sporadic by 2010, beginning in 2013, outbreaks occurred in the western and central highland areas of Vietnam, which prompted our study (4).

\section{The Study}

During June 2015-April 2018, the Pasteur Institute in Nha Trang, Vietnam, and the provincial health authority investigated 46 cases involving patients with

Author affiliations: Nagasaki University, Nagasaki, Japan

(N. Kitamura, K. Yoshihara, L.-M. Yoshida); Pasteur Institute in Nha Trang, Nha Trang, Vietnam (T.T.T. Le, L.T. Le, L.D. Nguyen, A.T. Dao, T.T. Hoang, T.M. The, H.M. Do, H.X. Le, H.T. Do, M.Q. Vien); World Health Organization Representative Office for Vietnam, EPI, Hanoi, Vietnam (M. lijima); National Institute of Hygiene and Epidemiology, Hanoi (A.D. Dang)

DOI: https://doi.org/10.3201/eid2603.191027 suspected diphtheria, 8 of whom died, and 49 asymptomatic contacts in the provinces of Quang Nam and Quang Ngai in the central highlands region of Vietnam (Figure 1). We used standard case investigation forms to collect demographic and clinical information. We collected throat swab specimens from 93 patients and contacts but were unable to collect samples from 2 patients who had died. No cutaneous diphtheria was reported.

We used sheep blood agar and tellurite medium cultures to identify Corynebacterium diphtheriae and extracted DNA with a QIAGEN DNA Mini Kit (QIAGEN, https://www.qiagen.com), following a standard protocol. We used 2 sets of primers, Tox1/Tox2 and Dipht6F/Dipht6R, for PCR testing (5). The Elek test for diphtheria is not available in Vietnam.

Laboratory testing confirmed diphtheria in 22 of 46 suspected cases: 17 patients, including 4 who died, tested positive in both culture and PCR tests, whereas 5 patients, including 1 who died, tested positive only by PCR. We categorized diagnosis as epidemiologic for 10 patients for whom PCR results were not available, 7 suspected cases and 3 in which the person died. We confirmed 2 of 49 asymptomatic contacts as carriers of diphtheria (6).

We used Api Coryne (bioMérieux, https://www. biomerieux.com) to identify biotypes of $C$. diphtheriae isolates; 15 of 17 culture-positive isolates were biotype mitis and 1 each was gravis and intermedius. We conducted multilocus sequence typing (MLST) by using 7 primer sets for $C$. diphtheriae housekeeping genes according to reported protocol (7). Using the C. diphtheriae MLST database (https://pubmlst.org/cdiphtheriae), we detected 4 sequence types (STs): ST67 $(n=7)$, ST209 $(n=9)$, ST243 $(n=7)$, and ST244 $(n=1)$.

Among the 31 patients with confirmed or suspected diphtheria, 21 (60\%) were male; age range was 1-45 years (median 10 years). We summarized case 

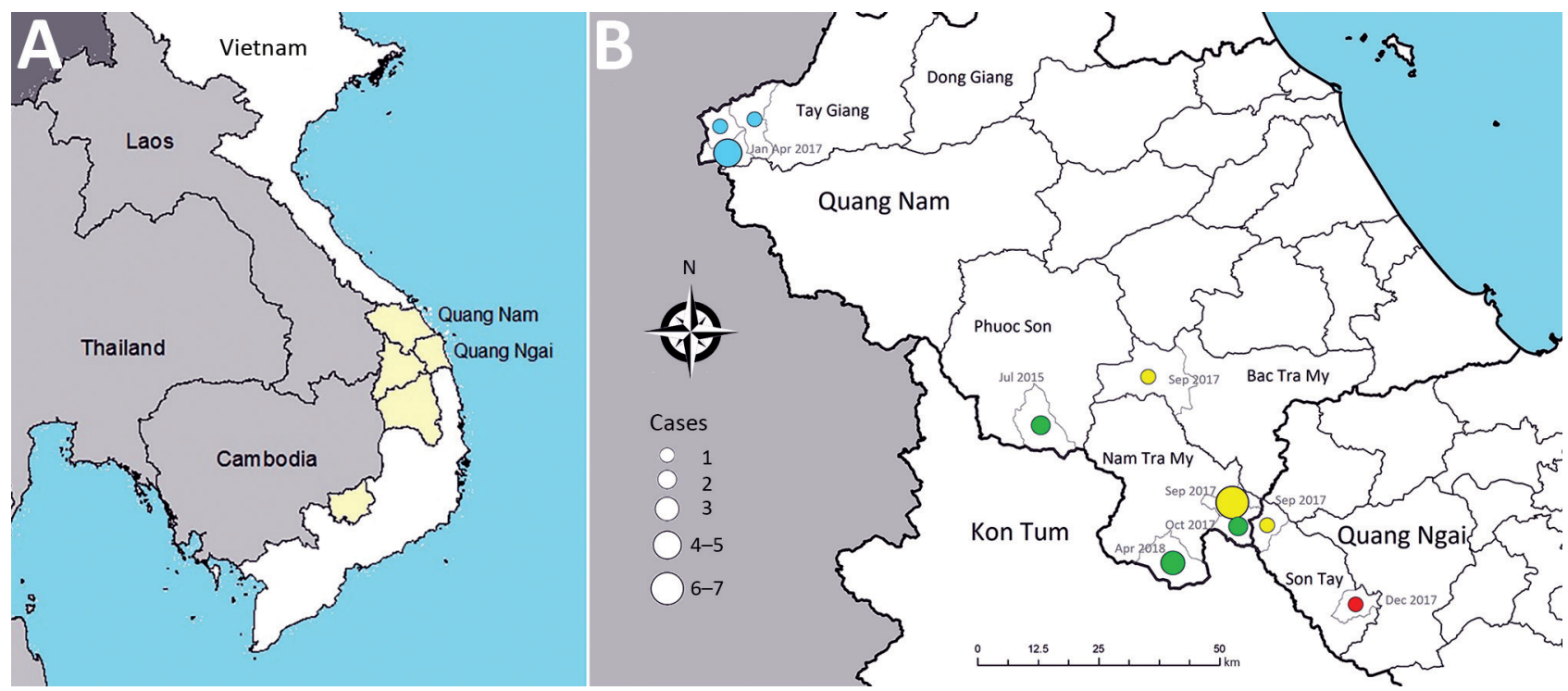

Figure 1. A) Provinces where diphtheria cases were identified in Vietnam in 2010s. Diphtheria cases were reported from provinces (shaded) neighboring Laos or Cambodia. B) Laboratory-confirmed diphtheria cases in the central highlands region of Quang Nam Province and Quang Ngai Province, central Vietnam, 2015-2018. Colored circles indicate separate outbreaks. Source: https://gadm.org/ download_country_v3.html

characteristics (Table 1) and epidemiologic links and STs by cluster (Table 2). The most common symptoms recorded were fever $(82 \%)$, followed by pseudomembrane and difficulty swallowing (76\%).

We determined geographic areas in which cases were identified (Figure 1). Most residents in the central highlands area were in ethnic minority groups.
Healthcare access is limited because of mountainous terrain and social barriers. In this area, each commune has a primary and a secondary school, but 10 communes share 1 district-level high school. All students, from primary through high school, live in dormitories during the week, and 30-50 students might live in $\mathrm{a} \approx 50 \mathrm{~m}^{2}$ room.

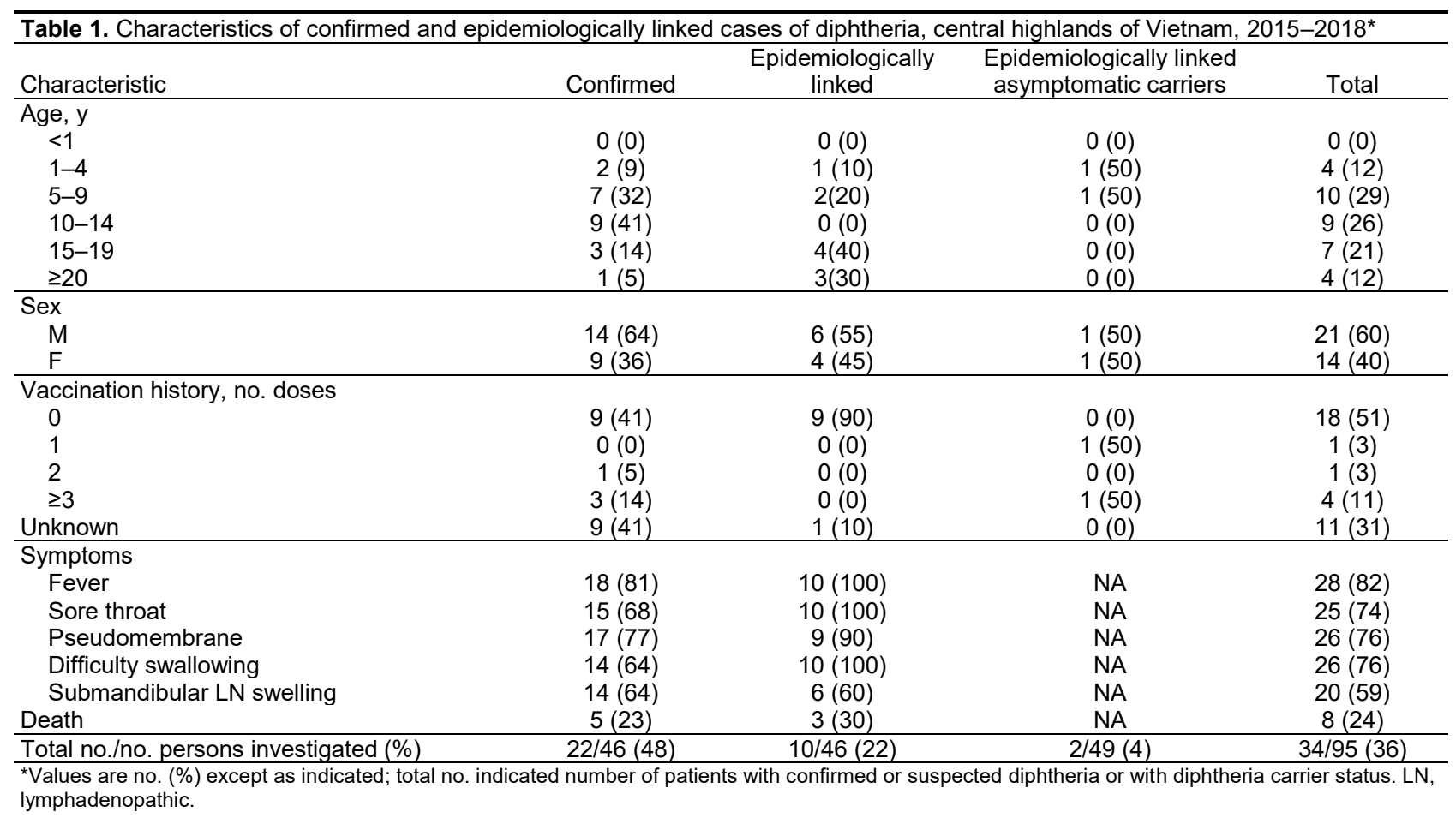


Table 2. Epidemiologic link and MLST results for 34 confirmed or epidemiologically linked case-patients with diphtheria, central highlands of Vietnam, 2015-2018*

\begin{tabular}{|c|c|c|c|c|c|c|c|c|c|c|}
\hline District & $\begin{array}{c}\text { Date of } \\
\text { symptom onset }\end{array}$ & $\begin{array}{l}\text { Patient } \\
\text { age, y/sex }\end{array}$ & Epidemiologic link & $\begin{array}{l}\text { Vaccine } \\
\text { status } †\end{array}$ & Died & $\begin{array}{c}\text { Culture } \\
\text { result }\end{array}$ & PCR & ST & Biotype & Case \\
\hline \multirow[t]{11}{*}{ Phuoc Son } & 2015 Jun 30 & $26 / F$ & Patient 1 & UNK & $\mathrm{X}$ & $\neq$ & $\neq$ & & & Linked \\
\hline & 2015 Jun 30 & $18 / \mathrm{M}$ & & UNK & & - & $\S$ & & & Linked \\
\hline & 2015 Jul 4 & $17 / F$ & & UNK & $\mathrm{X}$ & - & $\S$ & & & Linked \\
\hline & $2015 \mathrm{Jul} 4$ & $27 / M$ & Patient 1's husband & UNK & & + & $+\S$ & 67 & mitis & Confirmed \\
\hline & 2015 Jul 4 & $16 / \mathrm{M}$ & & UNK & & - & $\S$ & & & Linked \\
\hline & 2015 Jul 5 & 7/M & Patient 1's son & UNK & & - & $\S$ & & & Linked \\
\hline & 2015 Jul 5 & 20/M & & UNK & & - & $\S$ & & & Linked \\
\hline & 2015 Jul 8 & $45 / \mathrm{M}$ & & UNK & & - & $\S$ & & & Linked \\
\hline & 2015 Jul 9 & $1 / F$ & & UNK & & - & $\S$ & & & Linked \\
\hline & 2015 Jul 14 & $14 / \mathrm{M}$ & & UNK & & + & $+\S$ & 67 & mitis & Confirmed \\
\hline & 2015 Jul 14 & $9 / \mathrm{F}$ & & UNK & & - & $\S$ & & & Linked \\
\hline \multirow[t]{2}{*}{ Tay Giang } & 2017 Jan 10 & $16 / \mathrm{M}$ & Tay Giang HS & UNK & $\mathrm{X}$ & $\neq$ & $\neq$ & & & Linked \\
\hline & 2017 Jan 10 & $17 / \mathrm{M}$ & & UNK & $\mathrm{X}$ & + & + & 243 & mitis & Confirmed \\
\hline Son Tay & 2017 Mar 15 & $13 / \mathrm{M}$ & & 3 & $\mathrm{X}$ & + & + & 209 & mitis & Confirmed \\
\hline \multirow[t]{6}{*}{ Tay Giang } & 2017 Apr 20 & $7 / \mathrm{M}$ & & 4 & $\mathrm{X}$ & + & + & 243 & mitis & Confirmed \\
\hline & 2017 Apr 22 & $15 / F$ & & UNK & & + & + & 243 & mitis & Confirmed \\
\hline & 2017 Apr 25 & $7 / M$ & Gari PS & UNK & & + & + & 243 & mitis & Confirmed \\
\hline & 2017 May 20 & $10 / \mathrm{M}$ & Patient 2 (Gari SS) & UNK & & + & + & 243 & mitis & Confirmed \\
\hline & 2017 May 20 & $10 / \mathrm{M}$ & Gari SS & 3 & & + & + & 243 & mitis & Confirmed \\
\hline & 2017 May 23 & $15 / M$ & $\begin{array}{l}\text { Patient 2's } \\
\text { brother's friend }\end{array}$ & 0 & & + & + & 243 & mitis & Confirmed \\
\hline Bac Tra My & 2017 Sep 5 & $5 / F$ & & UNK & & - & + & 209 & & Confirmed \\
\hline \multirow[t]{7}{*}{ Nam Tra My } & 2017 Sep 27 & $12 / \mathrm{M}$ & Tra Van SS & UNK & & + & + & 209 & mitis & Confirmed \\
\hline & 2017 Sep 27 & $8 / \mathrm{M}$ & Tra Van PS & 0 & $\mathrm{X}$ & + & + & 209 & mitis & Confirmed \\
\hline & 2017 Sep 30 & $9 / F$ & & 0 & & - & + & 209 & & Confirmed \\
\hline & 2017 Sep 30 & $10 / F$ & & 0 & & - & + & 209 & & Confirmed \\
\hline & 2017 Sep 30 & $8 / \mathrm{F}$ & & 0 & & - & + & 209 & & Confirmed \\
\hline & 2017 Oct 3 & $11 / \mathrm{F}$ & & 0 & & + & + & 209 & mitis & Confirmed \\
\hline & 2017 Oct 3 & $10 / \mathrm{M}$ & & 0 & & + & + & 209 & mitis & Confirmed \\
\hline \multirow[t]{2}{*}{ Nam Tra My } & 2017 Oct 8 & $12 / \mathrm{F}$ & Tra Vinh SS & UNK & & - & + & 67 & & Confirmed \\
\hline & 2017 Oct 12 & $13 / \mathrm{M}$ & & UNK & & + & + & 67 & mitis & Confirmed \\
\hline Son Tay & 2017 Dec 24 & $3 / \mathrm{F}$ & & UNK & & + & + & 244 & gravis & Confirmed \\
\hline \multirow[t]{3}{*}{ Nam Tra My } & 2018 Apr 17 & $4 / \mathrm{M}$ & Man Di NS & 2 & $\mathrm{X}$ & - & + & 67 & intermed & Confirmed \\
\hline & 2018 Apr 24 & 4/M & & $3+1 S I A$ & & + & + & 67 & & Linked \\
\hline & 2018 Apr 24 & $5 / F$ & & $1+1 \mathrm{SIA}$ & & - & + & 67 & & Linked \\
\hline \multicolumn{11}{|c|}{ 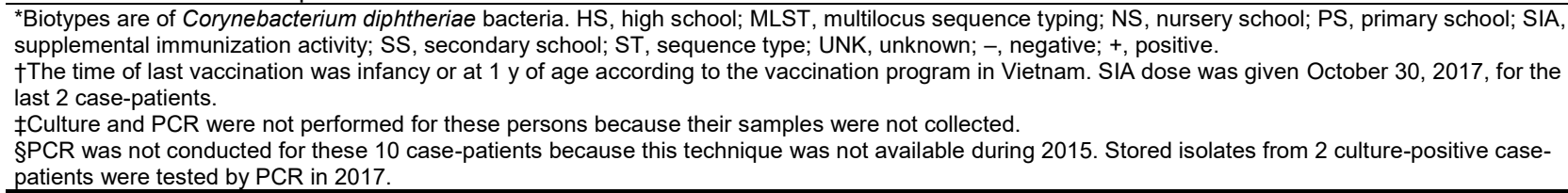 } \\
\hline
\end{tabular}

After January 2017, in each commune, diphtheria clusters formed mainly by school; cases in each school-based cluster shared the same ST. School clusters of the same ST in 2 communes in Tay Giang District were linked by a student who commuted between the communes. We could not identify any other epidemiologic links between clusters. An epidemic curve (Figure 2) showed the ST and outcome of cases by their onset. A long gap between clusters might indicate that the disease was transmitted through asymptomatic or skin carriers. However, further genomic testing is necessary to clarify the transmission pathway.

Of 8 persons who died, 3 were vaccinated, 1 each with 2, 3, and 4 doses. However, the vaccination history of $85 \%$ of patients was unknown. To compensate for the lack of vaccination history, we obtained administrative details of vaccination coverage in Nam Tra My District during 2013-2016. Of the 10 communes, only 3 (Tra Van, Tra Vinh, and Tra Nam) reported cases. We compared the ratios of vaccinated and unvaccinated children and found a significantly smaller proportion of children had received DTP3 in the outbreak communes than in nonoutbreak communes $(57 \%$ [95\% CI 53.3\%$61.2 \%]$ vs. $77 \%$ [ $95 \%$ CI $87.0 \%-90.1 \%] ; p<0.05$ by $\chi^{2}$ test).

\section{Conclusions}

Our investigation detected 22 patients with laboratory-confirmed C. diphtheriae cases during 2015-2018 in this region of Vietnam, $83 \%$ of whom were $>5$ 


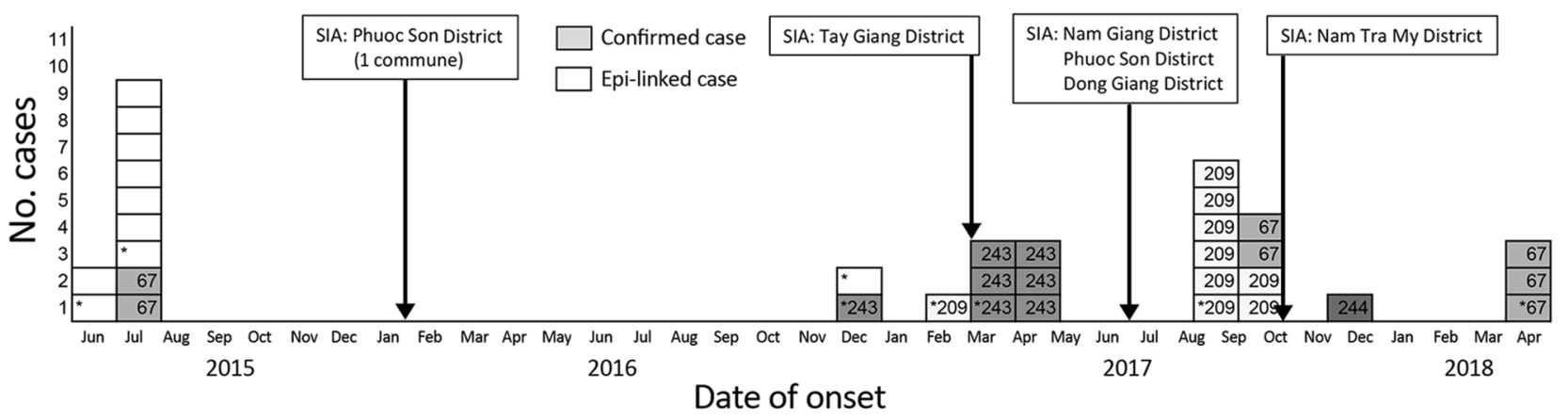

Figure 2. Confirmed and probable cases of diphtheria identified during June 2015-April 2018 in Vietnam. Numbers indicate multilocus sequencing type of confirmed cases with sequence types (STs) ST67, ST 243, ST209, and ST244 (gray shading). White indicates epidemiologically linked cases, and asterisks indicate cases in which the patient died. Epi, epidemiologically; SIA, supplemental immunization activity.

years of age. It has been predicted that age of diphtheria case-patients could increase after introduction of DTP because a high proportion of older persons will be susceptible to the disease due to reduced circulation of bacteria, especially when no booster dose is provided (8). The 4 MLST types identified in this study (ST67, ST209, ST243, and ST244) were also identified in Thailand, Cambodia, the Philippines, and Binh Phuoc Province in Vietnam in the 2010s $(4,9,10)$. We found only 1 ST in each cluster location, which might indicate 1 person as the source of infection in each location. In addition, we identified no clear epidemiologic link among clusters. Detecting different STs between clusters indicates that multiple strains of $C$. diphtheriae were circulating in Vietnam, as well as in neighboring countries. This transmission pattern might not have changed since the prevaccination era when diphtheria was reported to spread from school to school or neighborhood to neighborhood (11).

The reemergence of diphtheria in Vietnam raises several concerns. Administrative coverage, although not always accurate, indicated DTP3 coverage of $57 \%$, possibly creating a larger pool of susceptible people. In 2013, the health service temporarily suspended DTP immunization during a severe adverse event case investigation, which halved DTP3 coverage in the country (2) and potentially led to outbreaks. Students also share crowded school dormitories, which is a major factor for spreading disease. Moreover, students go home on weekends, increasing the chance of transmission between their schools and homes. Our finding of vaccinated people dying is particularly alarming because it might indicate a waning of vaccine-derived immunity.

Several interventions were conducted to control outbreaks. Erythromycin tablets were distributed to all contacts of diphtheria patients. However, only 2 asymptomatic carriers were identified among 49 contacts, lower than expected considering that $97 \%$ of case-patients could be asymptomatic in a vaccinated population (12). However, the sensitivity of laboratory testing might have been low because of the length of time required to collect and transport samples or because of prior antimicrobial drug use, so some carriers likely were not identified.

Supplemental immunization activities were conducted in the outbreak area and 2 neighboring districts (Nam Giang and Dong Giang). Healthcare agencies initiated 2 campaigns: the first, targeting persons 5-40 years of age, sought to administer 3 doses of tetanus-diphtheria vaccine and achieved $>90 \%$ coverage. Simultaneously, a second campaign was conducted to administer DPT to previously unvaccinated children 1-4 years of age. However, 1 unvaccinated person with diphtheria and 2 asymptomatic carriers who had received 1 dose of DPT were reported 6 months after the supplemental immunization activity. This finding was probably because diphtheria toxoid vaccine does not prevent transmission but prevents respiratory disease (13); thus, carriage of the organism persists.

Although Vietnam has maintained high DTP3 coverage nationally, efforts should be intensified to increase coverage in specific areas of the country (14). Persistent immunity resulting from DTP3 alone is not apparent (14), and immunity might wane before children start school (15). The World Health Organization recommends that students receive a booster vaccination when entering school (15). However, even if this recommendation is adopted, maintaining high uptake of primary and booster doses remains critical. 


\section{Acknowledgments}

We thank staff who were designated to the infectious disease surveillance program in central Vietnam for collecting and summarizing information, and healthcare workers at the Pasteur Institute in Nha Trang and Quang Nam Provincial Health Services for conducting multiple investigations.

This study was partly supported by Japan Agency for Medical Research and Development (grant no.

JP19fm0108001: Japan Initiative for Global Research Network on Infectious Diseases) for research activities of Nagasaki University in Vietnam and the Joint Usage/ Research Center on Tropical Disease, Institute of Tropical Medicine, Nagasaki University.

\section{About the Author}

Dr. Kitamura is an assistant professor in the Department of Pediatric Infectious Diseases, Institute of Tropical Medicine, Nagasaki University, Nagasaki, Japan, who has been stationed at the Pasteur Institute in Nha Trang, Vietnam, since 2016. Her primary research interest is epidemiology and control of infectious diseases, especially vaccine-preventable diseases.

\section{References}

1. Hardy IR, Sutter RW, Dittmann S. Current situation and control strategies for resurgence of diphtheria in newly independent states of the former Soviet Union. Lancet. 1996;347:1739-44. https:/ / doi.org/10.1016/ S0140-6736(96)90811-9

2. Immunization, vaccines and biologicals: data, statistics and graphics. Geneva: World Health Organization; 2019 [cited 2020 Jan 18]. http:/ / www.who.int/immunization/ monitoring_surveillance/data

3. Ministry of Health Expanded Program of Immunization. 25 years of expanded program of immunization in Vietnam. Hanoi (Vietnam): The Ministry; 2012.

4. Vo TT, Phan VT, Pham TH, Ho NL, Pham DQ, Nguyen VT, et al. Surveillance of Corynebacterium diphtheriae-caused diphtheria in Binh Phuoc province, June 2016 [in Vietnamese]. Vietnam Journal of Preventive Medicine. 2017;2017:11.

5. Nakao H, Popovic T. Development of a direct PCR assay for detection of the diphtheria toxin gene. J Clin Microbiol.
1997;35:1651-5. https:// doi.org/10.1128/JCM.35.7. 1651-1655.1997

6. Immunization, vaccines and biologicals: vaccine preventable disease surveillance standard. Geneva: World Health Organization; 2018 [cited 2020 Jan 19]. https://www.who.int/immunization/monitoring surveillance/burden/vpd/standards

7. Bolt F, Cassiday P, Tondella ML, Dezoysa A, Efstratiou A, Sing A, et al. Multilocus sequence typing identifies evidence for recombination and two distinct lineages of Corynebacterium diphtheriae. J Clin Microbiol. 2010;48:4177-85. https://doi.org/10.1128/JCM.00274-10

8. Clarke KE, MacNeil A, Hadler S, Scott C, Tiwari TS, Cherian T. Global epidemiology of diphtheria, 2000-2017. Emerg Infect Dis. 2019;25:1834-42. https:/ / doi.org/10.3201/ eid2510.190271

9. Pailin P, Sittisak S, Pimrat K, Junti K, Jivapaisarnpong T, Paveenkittiporn $W$, et al. Epidemiological and serological study of re-emerging diphtheria in Dansai District, Loei Province, Thailand, June to October 2012. Surveillance and Investigation Reports. 2015;8:13-21.

10. Doyle CJ, Mazins A, Graham RMA, Fang NX, Smith HV, Jennison AV. Sequence analysis of toxin gene-bearing Corynebacterium diphtheriae strains, Australia. Emerg Infect Dis. 2017;23:105-7. https://doi.org/10.3201/ eid2301.160584

11. Doull JA, Lara H. The epidemiological importance of diphtheria carriers. Am J Epidemiol. 1925;5:508-29. https:/ / doi.org/10.1093/oxfordjournals.aje.a119677

12. Miller LW, Older JJ, Drake J, Zimmerman S. Diphtheria immunization. Effect upon carriers and the control of outbreaks. Am J Dis Child. 1972;123:197-9. https://doi.org/ 10.1001/archpedi.1972.02110090067004

13. Plotkin S, Orenstein W, Offit P, Edwards KM, editors. Vaccines, 7th ed. Philadelphia: Elsevier; 2018.

14. Immunization, vaccines and biologicals: the immunological basis for immunization series module 2: diphtheria. Geneva: World Health Organization; 2009 [cited 2020 Jan 24]. https://www.who.int/immunization/documents/ ISBN9789241597869

15. World Health Organization. Diphtheria vaccine: WHO position paper, August 2017 - recommendations. Vaccine. 2018; 36:199-201. https:/ / doi.org/10.1016/ j.vaccine.2017.08.024

\footnotetext{
Address for correspondence: Lay-Myint Yoshida, Nagasaki University, Institute of Tropical Medicine, Department of Pediatric Infectious Diseases, 1-12-4 Sakamoto, Nagasaki-City, Nagasaki 852-8523, Japan; email: lmyoshi@nagasaki-u.ac.jp
} 